\title{
Surgical treatment of complex traumatic frontobasal lesions: personal experience in 74 patients
}

\author{
JÜRgen PIEK, M.D. \\ Neurosurgical Clinic, Ernst-Moritz-Arndt University, Greifswald, Germany.
}

\begin{abstract}
Object. The author's personal series included 148 patients who sustained traumatic frontobasal injuries and were treated between 1986 and 1999. Included in this study are 74 of 148 patients with acute injuries and complex frontobasal lesions involving the frontal sinus, the cribriform/ethmoid roof complex, one or both orbital roofs, and the planum sphenoidale.

Methods. Surgery was delayed for up to 4 weeks postinjury in most patients (67 cases), whereas 17 with space-occupying hematomas and perforating injuries required early surgery. In 30 patients additional surgery was required to treat maxillofacial fractures, which was performed as a one-stage procedure together with the neurosurgical operation. The author performed a standard bifrontal craniotomy in which an intradural or combined intradural-extradural approach was used in all cases.

Four patients developed ascending meningitis in the preoperative period. As a result of surgical treatment one patient died, another two patients suffered from permanent defects, and three suffered from transitory neurological worsening. In two patients recurrence of a cerebrospinal fluid fistula occurred within a 3-month period posttreatment but was successfully obliterated during reoperation.

In the author's experience the intradural approach is comparable in terms of the morbidity, mortality, and success rates with extracranial approaches; additionally the intradural approach provides full visualization of the intracranial lesion. Useful olfactory nerve function can only be preserved if both olfactory nerves are left intact and not crushed during initial injury; this occurred in only five patients in this series.

Conclusions. If possible, surgical treatment of more complex lesions should be delayed until the 2nd or 3rd week following traumatic injury. With antibiotic prophylaxis the risk that ascending meningitis will occur prior to surgery is low. If the patient is systemically stable and brain swelling has resolved, even extensive one-stage neurosurgical/ maxillofacial procedures are well tolerated.
\end{abstract}

KEY WORDS - cerebrospinal fluid leak • head injury • maxillofacial fracture

Injuries to the anterior skull base frequently occur following head injuries. The main causes are high-speed motor vehicle and industrial accidents. Cerebrospinal fluid leaks occur in approximately 2 to $3 \%$ of all head-injured patients and in 11 to $12 \%$ of those who have sustained basal skull fractures. , $6,11,13,27-29,32,33$ In high-speed road accidents the incidence is even higher. ${ }^{13}$ Because the overall incidence of ascending meningitis in untreated cases may be as high as $85 \%$ (and the mortality rate as high as a $4.1 \%$ ) at 10 years follow up, surgical repair of the dura is usually indicated. ${ }^{14,19}$

The anterior skull base can be divided into a medial part contiguous with the nose and paranasal sinuses ("rhinobase" ${ }^{43,47}$ ) and a lateral portion including the orbital roofs and the lesser wings of the sphenoid. In cases in which the bone and dural defects only involve the posterior wall of the frontal sinus and areas of the skull base bordering on the sphenoid and ethmoid sinuses, rhinosurgical proce-

Abbreviations used in this paper: $\mathrm{CSF}=\mathrm{CT}=$ cerebrospinal fluid; computerized tomography; GCS = Glasgow Coma Scale; MMA = methylmethacrylate. dures (open or endoscopic surgery) have become increasingly popular because of their success rate and reduced morbidity rate compared with craniotomy. $1,5,8-10,15,22,31,34,39$

Complex fractures of the anterior skull are defined as through-and-through fractures of the frontal sinus with additional fractures of the cribriform plate/ethmoid-roof complex, of one or both orbital roofs, and the planum sphenoidale. According to Samii and Draf ${ }^{43}$ such lesions require the classical neurosurgical ${ }^{6,7,17}$ bifrontal intradural approach ${ }^{41}$ because they are almost always accompanied by extensive dural tearing and cerebral injury. ${ }^{37}$ Based on the personal experience in treating 74 cases of such complex traumatic lesions of the anterior skull base, the author presents indications, surgical strategy and approaches, and clinical results.

\section{CLINICAL MATERIAL AND METHODS}

\section{Patient Population}

The author's personal series includes 148 patients with trauma-induced lesions of the anterior skull base. Fortyone of these patients sustained circumscribed traumatic le- 
TABLE 1

Clinical characteristics obtained in 74 patients

\begin{tabular}{cc}
\hline \hline Characteristic & No. of Patients \\
\hline neurological condition & \\
admission GCS score & \\
$3-8$ & 43 \\
$9-12$ & 24 \\
$13-15$ & 7 \\
preop GCS score & \\
$6-8$ & 10 \\
$9-12$ & 37 \\
$13-15$ & 27 \\
cause of injury & 48 \\
traffic accident & 15 \\
fall (total)* & 4 \\
gunshot wound $\dagger$ & 7 \\
other & 68 \\
bone fractures & 72 \\
frontal bone & 53 \\
cribriform/ethmoid complex & 42 \\
sphenoid & 30 \\
orbital roof & 27 \\
maxillofacial injury & 42 \\
involved cranial nerves & 2 \\
olfactory (one) & 3 \\
olfactory (both) & 2 \\
optic & \\
oculomotor Nerve & \\
other & \\
\hline
\end{tabular}

* Eight patients fell from heights, five from stairs, and two from horses. $\dagger$ Gunshot wounds were sustained from suicide attempt (two cases) and assault (two cases).

sions of the medial portion of the anterior skull base. Another 28 patients underwent surgery for recurrent CSF rhinorrhea, and an additional five patients underwent surgery for late infectious complications related to earlier injuries. The remaining 74 patients with complex traumatic lesions, as described in the introduction, are presented in this series. Each was prospectively evaluated. Details of the patient group are given in Table 1 and Fig. 1. The male/female ratio was approximately $6: 1$. Most of the injuries were caused by traffic accidents ( 48 cases), 15 were caused by falls, and four patients suffered gunshot wounds (two assaults, two suicide attempts). Rare causes were assaults in which other weapons such as a hammer or ax were used. All patients were admitted directly after the injury. Forty-three had sustained severe (GCS score $<9$ ), 24 had moderate (GCS score of 9-12), and seven patients a mild head injury. Thirty patients sustained additional maxillofacial injuries requiring operative treatment.

Operative repair of the defect was delayed in 57 patients. Seventeen patients underwent acute surgery, either for removal of space-occupying hematomas (three cases) or for a direct open head injury (14 cases). Most patients in whom surgery was delayed had recovered until their operation. Subsequently the GCS scores at the time of surgery were 13 to 15 in 27 cases, 9 to 12 in 37 cases, and 6 to 8 in 10 patients. Follow-up evaluation was performed at least 3 months postinjury.

\section{Diagnostic Procedures}

Thin-slice CT scans ${ }^{46}$ and plain skull $\mathrm{x}$-ray films were

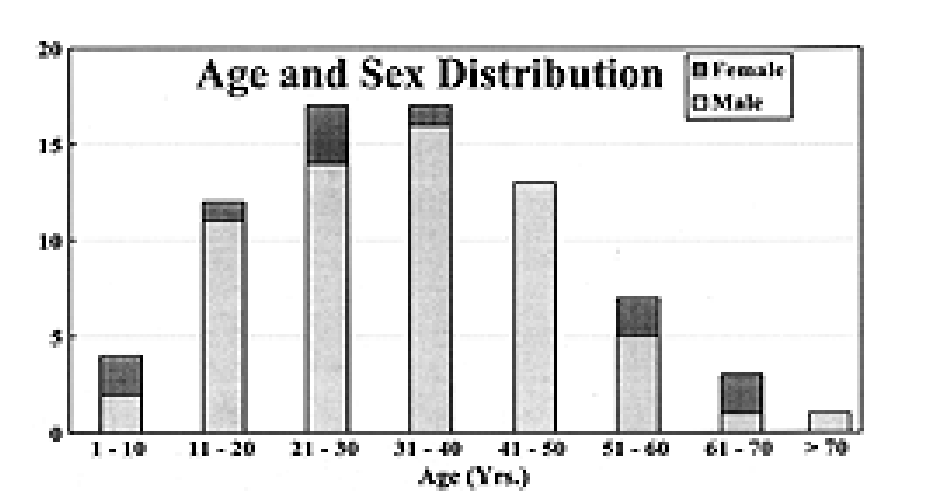

Fig. 1. Bar graph depicting the age and sex distribution of the patient group.

obtained in all patients. Additional diagnostic procedures used to demonstrate hidden fistulas included intrathecal metrizamide CT scanning, ${ }^{12}$ magnetic resonance imaging cisternography, ${ }^{20,21}$ and radionuclide cisternography ${ }^{3}$ in selected patients.

\section{Operative Treatment}

Early and Delayed Surgery With Antibiotic Medication. Seventeen patients required immediate surgical treatment directly after admission. Depending on the clinical state and on the presence of associated intra- and extracranial lesions, surgery was delayed for up to 4 weeks in the remaining 57 cases. In 30 patients associated maxillofacial fractures required additional surgical intervention. All of these operations were performed as one-stage procedures (that is, reconstruction of bone defects, repair of the CSF leak, and correction and ostheosynthesis of midface fractures). In these cases one-stage repair of the defects was only performed if hemodynamic stability was demonstrated, there was an absence of signs of intra- or extracranial infection, a GCS motor score of at least 5 was demonstrated, and a CT scan revealed the absence of signs of raised intracranial pressure (third ventricle and basal cisterns not obliterated).

All patients received prophylactic antibiotic agents (ampicilline/oxacilline, first-generation cephalosporin until 1994, and second-generation cephalosporine thereafter) to prevent ascending meningitis. ${ }^{19}$ The antibiotic therapy was initiated on admission and stopped on the 3rd day after definitive closure of the defect.

Repair of the CSF Leak. In rare cases we had to use preexisting scalp wounds to perform "atypical" skin incisions and/or craniotomies. In the remaining patients we used a standard bifrontal craniotomy and performed bilateral intradural inspection. Dural tears were sutured as far as possible. In smaller defects, remaining CSF leaks were sealed using a double layer of free temporal muscle and fibrin glue: a free piece of temporal muscle was pressed into the dural tear and sealed with fibrin glue; another, larger piece of muscle covering the tear and its surrounding region was attached above. Under no circumstances did we use alloplastic material to close the dural tear.

Reconstruction of Bone Defects. Although linear fractures do not require reconstruction and remodelling of 


\section{Complex traumatic frontobasal lesions}

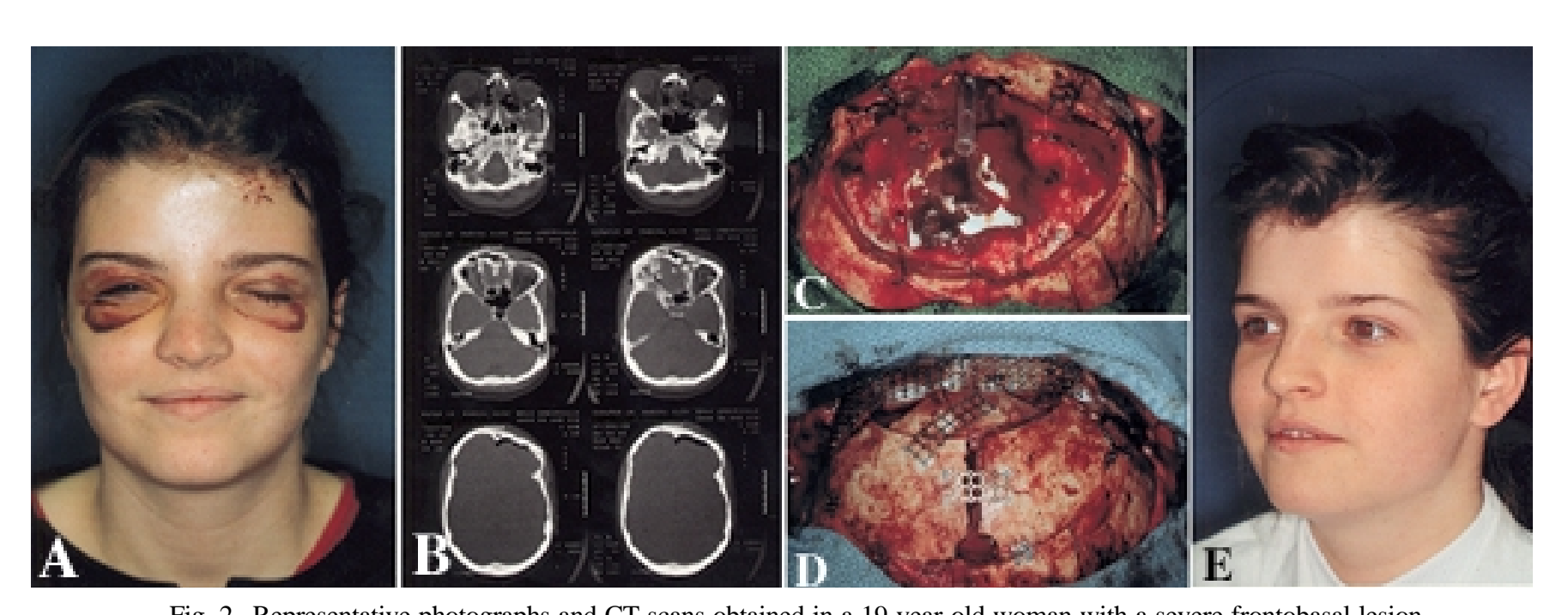

Fig. 2. Representative photographs and CI scans obtained in a 19-year-old woman with a severe trontobasal lesion involving the frontal bone, the frontal sinus, left orbital roof, planum ethmoidale, planum sphenoidale. Her admission GCS score was 12. Operative treatment was delayed until Day 11 posttrauma. A: Preoperative photograph. B: Initial CT scans revealing subdural air and bone injuries. $C$ : Intraoperative photograph obtained after bifrontal craniotomy, debridement, reconstruction of the frontal skull base, and closure of the dura. The frontal sinus was removed, and a transnasal, transethmoidal epidural drain was placed. $\quad D$ : Reconstruction of the frontal fractures by using multiple Vitallium mesh plates. E: Postoperative photograph obtained 2 years after surgery.

bone fragments, reconstruction is often required in more complex injuries. The bony cranial base was reconstructed, if large portions (two thirds) of the frontal skull base were destroyed. We used split calvarium in these cases.

In the majority of through-and-through fractures of the frontal sinus, the mucosa had been destroyed by the injury. Restoration of drainage of the frontal sinus through the frontonasal ducts in these cases is very unlikely, and cranialization of the frontal sinus is indicated. The posterior wall of the frontal sinus was drilled away, and all mucosa was removed. Adopting Röttgen's method, a transnasal, transethmoidal drain was placed through the region of the frontonasal ducts and left in place for approximately 2 weeks (see Fig. 2 C). This allows spontaneous canalization of the former frontal sinus cavity.

The contour of the frontal bone was either restored by previously cleansed fragments of the anterior and/or posterior wall, which were fixed with Vitallium (Howmedica Corp., Kiel, Germany) microplates and screws. In eight cases with large defects, we also used a Vitallium mesh for reconstruction of the orbital roof or the frontal bone by using a technique described elsewhere. ${ }^{36}$

\section{RESULTS}

One patient who was comatose on admission died as a direct result of the procedure. Due to extensive retraction of the right frontal lobe during immediate surgery, she developed bifrontal contusions. Two patients suffered from permanent and another three from transient neurological worsening also caused by extensive frontal lobe retraction.

Recurrence of the CSF leak was demonstrated in another two patients in whom operative revision was performed, and successful closure of the tear was achieved. One patient developed postoperative meningitis. Two patients suffered from preoperative meningitis while waiting for surgery. These patients were cured with high-dose antibiotic therapy.

\section{DISCUSSION}

The goal of neurosurgical management of frontobasal fractures is to prevent external deformity of the skull, seal the CSF leak, and to avoid chronic sinusitis. Long-term complications such as muco- or pyocele, meningitis, subdural empyema, and brain abscess should also be prevented. There is continuing debate regarding indications for surgery, timing of surgery, the best operative approach, and the material of choice with which to close dural leaks and bone defects. To cover all aspects each of these subjects would require a review article in itself. Only some points can be discussed in this paper.

The criteria for surgical intervention have been discussed extensively in a number of studies. ${ }^{13,16,18,24,26,29,30 \text {, }}$ 37,41 According to the authors of these studies, all acute patients underwent surgery for persistent CSF leaks, large comminuted fractures with a suspected dural tear, or perforating injuries.

\section{Goals and Timing of Surgery}

Complex fractures of the anterior skull base require a collaborative interdisciplinary approach. The neurosurgeon, the maxillofacial surgeon, the ear, nose, and throat surgeon, and sometimes the ophthalmologist are involved in the process of diagnosis, establishing the timing of necessary intervention(s), and deciding on the surgical approach. Cerebrospinal fluid fistulas resulting from circumscribed fractures of the medial portion of the anterior skull base can be sealed sufficiently via an extracranial rhinosurgical approach. ${ }^{1,5,8-10,15,22,31,34,39}$ In the present series, however, there was a negative selection of patients presenting with large fractures of the cranial base. 
Surgical treatment of such complex lesions has different aims: 1) closure of the CSF leak to prevent ascending meningitis; 2) reconstruction of cranial defects to maintain stability and to achieve comesis; 3 ) prevention of additional injuries to the brain and the cranial nerves; and 4) treatment of associated intracranial and maxillofacial lesions. All of these goals can be achieved only if a clear-cut diagnosis and an exact surgical plan are made. Proper diagnosis and surgical planning usually take time. Loew and coworkers ${ }^{29}$ have also shown that, with regard to outcome, delayed surgery is superior to immediate surgical intervention. According to these principles, immediate surgery was only performed in 17 of our patients. The indications for acute surgery included additional spaceoccupying intracranial hematomas and perforating injuries.

\section{Surgical Approach and Repair of the CSF Leak}

Determining the exact extent of the basal lesion helps in planning the surgical approach as well as choosing the best method for closing the dural and/or the bone defect. The ear, nose, and throat surgeon can manage circumscribed fractures by undertaking an extradural approach. . $^{1,5,8-10,15,22,24,25,30,31,34,35,39,42}$ Analysis of our results indicates that, with advanced neurosurgical techniques, an intracranial approach provides results even in complex lesions comparable with those obtained with extracranial approaches, and there is the additional advantage of full visualization of the intracranial lesion. ${ }^{38}$ We first approached all lesions intradurally. In 42 cases both olfactory nerves were found to be destroyed, which allowed additional extradural repair. In the remaining patients an intradural approach was performed to save olfactory nerve function. In only five of these patients (with both olfactory nerves anatomically intact and not crushed) normal olfactory function was noted postoperatively.

The best method of closing frontobasal defects is still a matter of debate, especially if there is a need for reconstructive surgery. There is no doubt that dural tears should be sutured whenever possible. In uncomplicated cases Samii and $\mathrm{Draf}^{42,43}$ prefer an inferior-based galeal pericranial flap. If more than two thirds of the anterior skull base is destroyed, they use a three-layer reconstruction of dura, pericranium, and an intermediate piece of MMA. Instead of dura the caudal layer can also consist of a rotated flap of the temporalis muscle. In a study by McCormack and

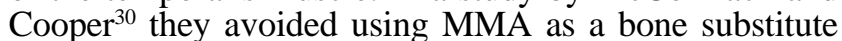
because of the risk of infection. Shaffrey, et al., ${ }^{45}$ have used rotated temporalis muscle and bone flaps, whereas Sekhar and Goel ${ }^{44}$ have indicated that they do not find an indication for bone reconstruction even in large defects, and they used rotated temporalis muscle flaps and inferiorly based pericranial flaps instead. If extensive defects of the orbital roof exist, Basso and coworkers ${ }^{2}$ prefer MMA, and Housepian ${ }^{23}$ has used a titanium mesh for reconstruction to avoid pulsation of the orbital contents. Based on the experience in 28 reoperations in which either inferiorly based pericranium flaps or free flaps had been used for the first operation, we do not feel that inferiorly based or rotated flaps are superior to free flaps. Because the blood supply to inferiorly based flaps is very limited, both types of flap tend to shrink. In our opinion it is more important to ensure that a flap is large enough to cover the leak more than $2 \mathrm{~cm}$ around its border to avoid recurrence of a fistula caused by shrinking. It should also be kept in mind that often rotation of the anterior part of the falx cerebri can successfully be used to close smaller dural defects.

Repair of frontal bone defects and of the anterior cranial fossa is often necessary in patients with large defects. In our opinion the material of choice is autologous bone, especially if larger parts of the frontal or sphenoid sinus are destroyed. We use split calvaria for this purpose. Use of split calvaria has the advantage of avoiding additional incision and is time saving compared with having to harvest bone from the iliac crest or from the ribs. Fixation is achieved using microscrews and plates.

\section{Defects of the Frontal and Sphenoidal Sinus}

In the majority of our patients both walls of the frontal sinus and the mucosa were destroyed. Linear fractures of the posterior wall may occur in less extensive injuries. If the fracture is only linear, the leak can be sealed by placing an inferiorly based pericranial flap to cover the posterior wall. In cases of complex fractures of the frontal sinus adaequate drainage of the frontal sinus and the ethmoid cell system is not generally observed. In these cases we perform complete cranialization of the frontal sinus (that is, the posterior wall is drilled away and the mucosa is completely removed). Adapting a method proposed by Röttgen (Fig. 2 C), we create an external transethmoidal drainage system: a large silicone tube is inserted into the epidural space by perforating the cribriform place from the nasal cavity. Outside the nose it is initially connected to a sterile surgical glove. If secretion stops, the glove is removed and the drainage system left in place for at least 2 weeks. Thus a fully epithelialized duct is created for permanent pneumatization of the ethmoidal cell system.

Isolated fractures of the sphenoid sinus were not observed in the present series. If we found the roof of the sphenoid sinus to be fractured and the sinus itself to be opened, we removed all mucosa, packed the sinus with muscle pieces and fibrin glue, and covered it with an additional free flap of temporal muscle. Linear fractures of the sphenoid are best managed by using a single layer cover of muscle.

\section{Surgery-Related Outcome}

In a large series reported by Eljamel and Foy ${ }^{18}$ in which 149 patients underwent surgery via an intracranial approach, the surgery-related mortality rate was $1.3 \%$ and the morbidity rate was $24.9 \%$. These results compare with ours.

Large CSF leaks caused by comminuted fractures require complete intraoperative exposure of the whole frontal skull base (including the roof of the sphenoid sinus). Using an extradural approach, this exposure is usually not possible without injuring the intact olfactory tracts. In our series, however, an intact olfactory tract on both sides was found intraoperatively in only five patients with such lesions. Complete exposure of the anterior skull base, however, requires some retraction of the frontal lobes even during microsurgical procedures. Lumbar drainage or direct puncture of the lateral ventricle may help to collapse the brain to avoid excessive retraction to some extent. 
Although these techniques were applied in all of our patients, one death, three transient deficits, and two permanent neurological deficits were observed. All but one of these patients underwent surgery between Days 10 and 14 following trauma. In retrospect we understand that some brain swelling was still present in these cases and that the operation should have been delayed for a longer period of time. One can also debate whether prolonged intracranial pressure monitoring may be helpful in these cases.

In the current series we present a selected series of cases from a larger series of head-injured patients. In follow-up evaluation we focused on the surgery-related results (for example, additional morbidity and mortality caused by the operation and recurrence of the CSF leak), not on the outcome of the brain injury itself.

As it is well known from the literature, occurence or recurrence of a CSF leak following traumatic injury may be observed even after decades after the original event. ${ }^{40}$ A follow-up study for this long period is not realistic. In our patients, we only considered early recurrence of a leak during the first 3 months. This was the case in two patients in whom reoperation successfully resolved the CSF leak.

\section{CONCLUSIONS}

Using clinical examination, skull radiography, and thinslice CT scanning, we can classify patients with frontobasal injuries into those with midline lesions in which rhinosurgical approaches may be indicated and those with complex frontobasal fractures involving the lateral portions of the anterior skull base. These patients are best treated via the "classic" bifrontal route with intradural inspection. Evaluation of our results shows that this intradural approach is comparable with other procedures in terms of surgery-related morbidity, mortality, and success rates, and in addition the intradural approach affords full visualization of the intracranial pathological entities and a low recurrence rate. When possible, surgical treatment of these complex lesions should be delayed until the 2 nd or 3rd week following trauma. The risk that the patient will develop ascending meningitis is negligible after initiation of antibiotic therapy until the time of surgery. If the patient is systemically stable and brain swelling has resolved, even extensive one-stage procedures are well tolerated.

\section{References}

1. Aboulker P, Lebaeau J, Sterkers JM: [Treatment of ethmoidofrontal meningeal fistulas. Apropos of 15 cases successfully operated by exocranial approach.] Ann Otolaryngol Chir Cervicofac 83:27-32, 1966 (Fr)

2. Basso A, Carizzo C, Kreutel A: Transcranial approach to lesions of the orbit, in Schmidek HH, Sweet WH (eds): Operative Neurosurgical Techniques. Philadelphia: WB Saunders, 1995, pp 205-236

3. Bliek AJ, Miller JD: RISA cisternography: a review of 29 cases. J Can Assoc Radiol 22:215-221, 1971

4. Brawley BW, Kelly WA: Treatment of skull fractures with and without cerebrospinal fistulae. J Neurosurg 26:57-61, 1967

5. Briant TDR, Snell D: Diagnosis of cerebro-spinal rhinorrhea and the rhinologic approach to its repair. Laryngoscope 77: 1390-1409, 1967

6. Brisman R, Hughes JE, Mount LA: Cerebrospinal fluid rhinorrhea. Arch Neurol 22:245-252, 1970
7. Cairns H: Injuries to the frontal and ethmoidal sinuses with special reference to cerebrospinal fluid rhinorrhea and aeroceles. J Laryngol 52:589-623, 1937

8. Calcaterra TC: Diagnosis and management of ethmoid cerebrospinal rhinorrhea. Otolaryngol Clin North Am 18:99-105, 1985

9. Calcaterra TC: Extracranial surgical repair of cerebrospinal rhinorrhea. Ann Otol Rhinol Laryngol 89:108-116, 1980

10. Calcaterra TC, Moseley JI, Rand RW: Cerebrospinal rhinorrhea: extracranial surgical repair. West J Med 127:279-283, 1977

11. Cantore GP, Delfini R, Gambacorta D, et al: Cranio-orbito-facial injuries: technical suggestions. J Trauma 19:370-375, 1979

12. Colquhoun IR: CT cisternography in the investigation of cerebrospinal fluid rhinorrhoea. Clin Radiol 47:403-408, 1993

13. Dagi TF, George ED: Surgical management of cranial cerebrospinal fluid fistulas, in Schmidek HH, Sweet WH (eds): Operative Neurosurgical Techniques. Philadelphia: WB Saunders, 1995, pp 117-131

14. Dagi TF, Meyer FB, Poletti CA: The incidence and prevention of meningitis after basilar skull fracture. Am J Emerg Med 3:295-298, 1983

15. Daly DT, Lydiatt WM, Ogren FP, et al: Extracranial approaches to the repair of cerebrospinal fluid rhinorrhea. Ear Nose Throat J 71:311-313, 1992

16. Dandy WE: Pneumocephalus (intracranial pneumatocele or aerocele). Arch Surg 12:949-982, 1926

17. Eden K: Traumatic cerebrospinal rhinorrhea: repair of the fistula by a transfrontal intradural operation. Br J Surg 290: 299-303, 1941

18. Eljamel MS, Foy PM: Post-traumatic CSF fistulae, the case for surgical repair. Br J Neurosurg 4:479-483, 1990

19. Eljamel MS, Foy PM: Acute traumatic CSF fistulae: the risk of intracranial infection. Br J Neurosurg 4:381-385, 1990

20. Eljamel MS, Pidgeon CN: Localization of inactive cerebrospinal fluid fistulas. J Neurosurg 83:795-798, 1995

21. Eljamel MS, Pidgeon CN, Toland J, et al: MRI cisternography, and the localization of CSF fistulae. Br J Neurosurg 8: 433-437, 1994

22. Hosemann W, Nitsche N, Rettinger G, et al: [Endonasal, endoscopically controlled repair of dura defects of the anterior skull base.] Laryngorhinootologie 70:115-119, 1991 (Ger)

23. Housepian EM: Surgical management of intraorbital tumours; in Schmidek HH, Sweet WH (eds): Operative Neurosurgical Techniques. Philadelphia: WB Saunders, 1995, pp 183-193

24. Hubbard JL, McDonald TJ, Pearson BW, et al: Spontaneous cerebrospinal fluid rhinorrhea: evolving concepts in diagnosis and surgical management based on the Mayo Clinic experience from 1970 through 1981. Neurosurgery 16:314-321, 1985

25. Hudson WR, Hughes LA: Cerebrospinal rhinorrhea: diagnosis and management. South Med J 68:1520-1523, 1975

26. Leech PJ, Paterson A: Conservative and operative management for cerebrospinal-fluid leakage after closed head injury. Lancet 1:1013-1016, 1973

27. Lewin W: Cerebrospinal fluid rhinorrhea in closed head injury. Br J Surg 42:1-18, 1954

28. Lewin W: Cerebrospinal fluid rhinorrhea in nonmissile head injuries. Clin Neurosurg 12:237-252, 1964

29. Loew F, Pertuiset B, Chaumier EE, et al: Traumatic, spontaneous and postoperative CSF rhinorrhea. Adv Tech Stand Neurosurg 11:169-207, 1984

30. McCormack B, Cooper PR: Traumatic cerebrospinal fluid fistulas, in Narayan RK, Wilberger JE, Povlishock JT (eds): Neurotrauma. New York: McGraw-Hill, 1996, pp 639-653

31. McCormack B, Cooper PR, Persky M: Extracranial repair of cerebrospinal fluid fistulas: technique and results in 37 patients. Neurosurgery 27:412-417, 1990

32. Mendizabal GR, Moreno BC, Flores CC: Cerebrospinal fluid 
fistula: frequency in head injuries. Rev Laryngol Otol Rhinol 113:423-425, 1992

33. Mincy JE: Post-traumatic CSF fistula of the frontal fossa. J Trauma 6:618-621, 1966

34. Minnigerode B: [On the technic of extradural rhinosurgical covering of liquor fistulae following frontobasal head injuries.] Monatsschr Ohrenheilkd Laryngorhinol 101:441-446, 1967 (Ger)

35. Montgomery WW: Surgery for cerebrospinal fluid rhinorrhea and otorrhea. Arch Otolaryngol 84:538-550, 1966

36. Piek J: [A simple technique for reconstruction of anterior and middle cranial base after palliative tumor resection.] Zentralbl Neurochir 58:61-65, 1997 (Ger)

37. Probst C: Neurosurgical treatment of traumatic frontobasal CSF fistulae in 300 patients (1967-1989). Acta Neurochir 106: 37-47, 1990

38. Probst $\mathrm{C}$, Tomaschett C: [The neurosurgical treatment of traumatic fronto-basal spinal fluid fistulas. 66 cases (1982-1986).] Aktuelle Traumatol 20:217-225, 1990 (Ger)

39. Ross IB, Colohan AR, Black MJ: Extracranial repair of cerebrospinal fluid rhinorrhea. Can J Neurol Sci 17:320-323, 1990

40. Russell T, Cummins BH: Cerebrospinal fluid rhinorrhea 34 years after trauma: a case report and review of the literature. Neurosurgery 15:705-706, 1984

41. Samii M, Draf W: [Indication and treatment of frontobasal rhinoliquorrhoea from the ENT-surgical and neurosurgical point of view.] Laryngol Rhinol Otol 57:689-697, 1978 (Fr)
42. Samii M, Draf W: Surgery of the Skull Base. Berlin: SpringerVerlag, 1989, pp 159-232

43. Samii M, Draf W: Surgery of the Skull Base. Berlin: SpringerVerlag, 1989, pp 126-153

44. Sekhar LN, Goel A. Anterior and middle cranial base lesions, in Apuzzo MLJ (ed): Brain Surgery: Complication Avoidance and Management. New York: Churchill Livingstone, 1993, pp 2175-2195

45. Shaffrey ME, Persing JA, Shaffrey CI. Craniofacial reconstruction, in Apuzzo MLJ (ed): Brain Surgery: Complication Avoidance and Management. New York: Churchill Livingstone, 1993, pp 1373-1398

46. Thiebot J, Laissy JP: [Radiologic diagnosis of post-traumatic cerebrospinal rhinorrhea.] Ann Radiol 34:56-59, 1991 (Fr)

47. Wüllstein HL: [Has the terminology for the definition of otorhinolaryngology a practical significance?] HNO 20:259-261, 1972 (Ger)

Manuscript received May 18, 2000.

Accepted in final form June 20, 2000.

Address reprint requests to: Jürgen Piek, M.D., Neurosurgical Clinic, Ernst-Moritz-Arndt University, Ferdinand-Sauerbruchstraße, D-17487 Greifswald, Germany email: piek-jue@ uni-greifswald.de. 